\title{
Vertex Models on Feynman Diagrams
}

\author{
D.A. Johnston \\ Dept. of Mathematics \\ Heriot-Watt University \\ Riccarton \\ Edinburgh, EH14 4AS, Scotland \\ and \\ P. Plecháč \\ Mathematical Institute \\ 24-29 St Giles' \\ Oxford \\ OX1 3LB
}

29th August 1997

\begin{abstract}
The statistical mechanics of spin models, such as the Ising or Potts models, on generic random graphs can be formulated economically by considering the $N \rightarrow 1$ limit of $N \times N$ Hermitian matrix models. In this paper we consider the $N \rightarrow 1$ limit in complex matrix models, which describes vertex models of different sorts living on random graphs. From the graph theoretic perspective one is using matrix model and field theory inspired methods to count various classes of directed graphs.

We also make some remarks on vertex models on planar random graphs (the $N \rightarrow \infty$ limit) where the resulting matrix models are not generally soluble using currently known methods. Nonetheless, some particular cases may be mapped onto known models and hence solved.
\end{abstract}




\section{Introduction}

In recent papers we have discussed various analytical and numerical aspects of an economical way of describing spin models living on "thin" (i.e. generic, non-planar) random graphs [1, 2]. In particular, one finds mean-field like behaviour for the spin models due to the locally-tree-like structure of the graphs. This approach is based on the simple observation [3] that the thin graphs appear as the Feynman diagrams in the perturbative expansion of matrix models when the $N \rightarrow 1$ (scalar) limit is taken. An alternative $N \rightarrow \infty$ limit is perhaps more familiar for matrix models in the context of two-dimensional gravity, where the resulting "fat" graphs are of interest because of their relation to surfaces and string worldsheets. This relation is lost in the thin graph case 1 but the models still merit attention, both from the point of view of investigating various decorated random graphs and as a way of accessing mean field behaviour without infinite range interactions or the boundary problems of genuine trees like the Bethe lattice.

The thin graph models are amenable to a saddle point solution, using large order/instanton methods from path integrals [4] for the ordinary integrals which appear in this case. Such saddle point methods in graph theory been independently derived from a probabilistic viewpoint by Whittle in [0]. The topic of the current paper has also been presaged by the same author in [6], where the main concern was understanding the statistics of random directed graphs using complex integrals rather than the real integrals of 11, 2, 5. W. We take a rather different tack here, where our aim is to investigate vertex models on random graphs in their own right, so the focus is on the matter living on the graphs rather than the graphs themselves.

In diagrammatic terms the effect of going from a Hermitian to a complex matrix model is to place arrows on the edges of the graphs that appear in the perturbative expansion, giving a directed graph. We take $\phi^{\dagger}$ to be the "head" of the arrow by convention. This is true whatever the value of $N$ so it still holds, in particular, for the $N \rightarrow 1$ limit. We can pick out the $2 n$th order in the perturbative expansion by carrying out a contour integral in the coupling constant in an exactly analogous manner to the path integral calculations in $\mid$. The general form of the partition function we are interested in for a vertex model on an ensemble of thin random graphs with $2 n$ vertices is thus

$$
Z_{n}(\{\Lambda\}) \times N_{n}=\frac{1}{2 \pi i} \oint \frac{d \lambda}{\lambda^{2 n+1}} \int \frac{d \phi^{\dagger} d \phi}{2 \pi} \exp \left[-\left(\frac{1}{2} \phi^{\dagger} \phi+P\left(\phi^{\dagger}, \phi, \lambda,\{\Lambda\}\right)\right)\right],
$$

where $\phi$ is complex, $\phi^{\dagger}$ is its conjugate, $P\left(\phi^{\dagger}, \phi, \lambda,\{\Lambda\}\right)$ is the potential that specifies the particular vertex model, $\lambda$ is the overall vertex coupling that will invariably be scaled out in the ensuing discussion and $\{\Lambda\}$ is the set of remaining couplings that can be tuned to search for critical behaviour. The prefactor $N_{n}$ counts the number of undecorated graphs in the class of graphs of interest and will generically grow factorially with the number of vertices. For $\phi^{3}$ (3-regular) random graphs, for instance, it is given by

$$
N_{n}=\left(\frac{1}{6}\right)^{2 n} \frac{(6 n-1) ! !}{(2 n) ! !} .
$$

It is well known that complex matrix models with potentials of the form $P\left(\left(\phi^{\dagger} \phi\right), \lambda,\{\Lambda\}\right)$ generate "chequered" surfaces in the $N \rightarrow \infty$ limit, where all the loops have an even number of edges [7]. In this case the critical behaviour turns out to be identical to that of a Hermitian model, as restricting oneself to combinations of $\phi^{\dagger} \phi$ effectively gives a Hermitian model in terms of $|\phi|$. Something similar occurs in the thin $N \rightarrow 1$ limit, as was noted already in [6]. When one considers models where the in-degree (number of arrows in) and out-degree (number of arrows out) at each vertex is the same, as would be the case for potentials of the form $P\left(\left(\phi^{\dagger} \phi\right), \lambda,\{\Lambda\}\right)$ the saddle point equations are singular in the original variables due to the radial degree of freedom 2 . Switching to polar co-ordinates eliminates the singularity and one recovers the critical behaviour of the equivalent real integrals.

The natural symmetry to preserve in the vertex models is arrow reversal, or complex conjugation. In the integral for the partition function in equ.(11) this corresponds to demanding a real integrand. We

\footnotetext{
${ }^{1}$ The surfaces that might be associated with the graphs are "infinite genus", with a maximal handle density.

${ }^{2}$ An identical situation occurs in the path integral for coupled anharmonic oscillators, where the large orders saddle point solution has a divergence at the radially symmetric point [8].
} 
now note that we are not obliged to restrict ourselves to potentials composed only of combinations of $\phi^{\dagger} \phi$ to maintain this symmetry, which allows us to look at a more general class of potentials and observe a much richer critical behaviour than that seen for $P\left(\left(\phi^{\dagger} \phi\right), \lambda,\{\Lambda\}\right)$. We shall see in the sequel that the resulting models play a similar role to the eight-vertex model on a square lattice in that they contain many other soluble models as special cases.

In the next two sections of the paper we discuss models on thin $\phi^{3}$ and $\phi^{4}$ graphs separately. In all cases we shall convert our models back to a real notation by making the substitution $\phi=x+i y, \phi^{\dagger}=x-i y$ $(x, y$ real $)$, as well as pre-emptively scaling out the overall vertex coupling $\lambda$ in the actions we discuss. As it is the critical behaviour of the matter that is of interest, which is determined by the saddle point equations for the "fields" $\left(\phi^{\dagger}, \phi\right)$, we shall concentrate exclusively on them, omitting the trivial saddle point in $\lambda$. We then move on to discuss vertex models on planar graphs (i.e. the $N \rightarrow \infty$ limit), where the resulting matrix models are not soluble in general, highlighting some cases where solutions are available by mapping onto previously solved models.

\section{Vertex Models on (Thin) $\phi^{3}$ Graphs}

On $\phi^{3}$ graphs we can have vertices with 3 inward or 3 outward pointing arrows, 2 arrows in and 1 out, or 2 arrows out and one in as shown in Fig.1. These correspond to terms of the form $\left(\phi^{\dagger}\right)^{3}, \phi^{3},\left(\phi^{\dagger}\right)^{2} \phi$ and $\phi^{\dagger}(\phi)^{2}$ respectively in $P\left(\phi^{\dagger}, \phi, \lambda,\{\Lambda\}\right)$. We can construct four possible linear combinations of these that are invariant under the complex conjugation symmetry, namely

$$
\begin{aligned}
& {\left[\left(\phi^{\dagger}\right)^{3}+\phi^{3}\right], \quad i\left[\left(\phi^{3}-\left(\phi^{\dagger}\right)^{3}\right]\right.} \\
& {\left[\left(\phi^{\dagger}\right)^{2} \phi+\phi^{2}\left(\phi^{\dagger}\right)\right], \quad i\left[\left(\phi^{\dagger}\right)^{2} \phi-\phi^{2}\left(\phi^{\dagger}\right)\right]}
\end{aligned}
$$

so the most general invariant action $S\left(=\frac{1}{2} \phi^{\dagger} \phi+P\left(\phi^{\dagger}, \phi, \lambda=1,\{\Lambda\}\right)\right)$ one can write down is

$$
S=\frac{1}{2}\left\{\phi^{\dagger} \phi-\frac{\alpha}{3}\left[\left(\phi^{\dagger}\right)^{3}+\phi^{3}\right]-i \frac{\beta}{3}\left[\left(\phi^{3}-\left(\phi^{\dagger}\right)^{3}\right]-\gamma\left[\left(\phi^{\dagger}\right)^{2} \phi+\phi^{2}\left(\phi^{\dagger}\right)\right]-i \delta\left[\left(\phi^{\dagger}\right)^{2} \phi-\phi^{2}\left(\phi^{\dagger}\right)\right]\right\}\right.
$$

or, grouping together the similar vertices

$$
S=\frac{1}{2}\left\{\phi^{\dagger} \phi-\frac{(\alpha-i \beta)}{3}\left(\phi^{\dagger}\right)^{3}-\frac{(\alpha+i \beta)}{3} \phi^{3}-(\gamma+i \delta)\left[\left(\phi^{\dagger}\right)^{2} \phi\right]-(\gamma-i \delta)\left[\phi^{2}\left(\phi^{\dagger}\right)\right]\right\} .
$$

We thus see it is natural to have the $\left(\phi^{\dagger}\right)^{3}, \phi^{3}$ and $\left(\phi^{\dagger}\right)^{2} \phi, \phi^{2}\left(\phi^{\dagger}\right)$ vertices appearing with complex (but conjugate) weights. We can cast this action in real form by writing $\phi=x+i y, \phi^{\dagger}=x-i y$, with $x, y$ real which gives

$$
S=\frac{1}{2}\left(x^{2}+y^{2}\right)-\frac{(\alpha+3 \gamma)}{3} x^{3}-\frac{(\beta+3 \delta)}{3} y^{3}-(\gamma-\alpha) x y^{2}-(\delta-\beta) x^{2} y .
$$

This transformation is rather reminiscent of the line decomposition that is used for vertex models on regular lattices [9], with $x$ being black and $y$ white lines, but we do not have line conservation at each vertex for the $\phi^{3}$ model. The saddle point equations $\partial S / \partial x, \partial S / \partial y=0$ for this action are explicitly soluble for general values of the couplings, but the solutions are not terribly illuminating for the purposes of extracting different critical behaviours when written down baldly.

We can get a little more insight by reducing various subsets of the coupling constant space to already known models. One obvious reduction is to set $\beta=\delta=0$ which removes the terms antisymmetric in $\left(\phi^{\dagger}, \phi\right)$ in the action. This leaves one with

$$
S=\frac{1}{2}\left(x^{2}+y^{2}\right)-\frac{(\alpha+3 \gamma)}{3} x^{3}-(\gamma-\alpha) x y^{2}
$$

which displays mean-field Ising critical behaviour on a suitable coupling constant locus. 
This can be seen by considering the standard action for the Ising model on $\phi^{3}$ graphs

$$
S=\frac{1}{2}\left(x^{2}+y^{2}\right)-c x y-\frac{1}{3}\left(x^{3}+y^{3}\right)
$$

(where $c=\exp (-2 \beta)$ ) whose Feynman diagram expansion represents Ising spins with Hamiltonian

$$
H=\beta \sum_{i j} \sigma_{i} \sigma_{j}
$$

living on the graphs, where the spin interactions are along nearest neighbour $\langle i j\rangle$ edges. If we carry out the linear transformation $x \rightarrow \frac{1}{\sqrt{2}}(x+y), y \rightarrow \frac{1}{\sqrt{2}}(x-y)$ and some judicious rescalings we obtain

$$
S=\frac{1}{2}\left(x^{2}+y^{2}\right)-\frac{1}{3}\left[x^{3}+3 c^{*} x y^{2}\right],
$$

where $c^{*}=(1-c) /(1+c)$, which is identical (up to a rescaling) with equ.(7), provided we identify $c^{*}$ with $(\gamma-\alpha) /(\alpha+3 \gamma)$.

The Ising model on $\phi^{3}$ graphs described by the action in equ.(8) displays a mean field transition at $c=1 / 3$, so the model in equ.(10) would be expected to display a transition at the "dual" value of $c^{*}=1 / 2$. A direct solution of the saddle point equations for equ. 10)

$$
\begin{aligned}
& x=1, \quad y=0 \quad\left(\text { Low } T^{*}\right) \\
& x=\frac{1}{2 c^{*}}, \quad y=\sqrt{\frac{2 c^{*}-1}{4\left(c^{*}\right)^{3}}}\left(\text { High } T^{*}\right),
\end{aligned}
$$

where the $T^{*}$ is the temperature associated with the dual coupling, $c^{*}=\exp \left(-2 \beta^{*}\right)$, reveals that this is indeed the case. It is worth remarking that the action of equ.(7), 10) on planar graphs (i.e. in the $N \rightarrow \infty$ limit) corresponds to an Ising model with spins situated at the vertices of a random triangulation dual to the original $\phi^{3}$ graph 10]. The notion of duality is not defined for the non-planar graphs of the $N \rightarrow 1$ limit but one could think of action representing spins residing on the vertices of the triangles in the cactus (or Husimi tree) diagram in Fig.2 interacting with their nearest neighbours. This cactus graph is the medial graph of the underlying $\phi^{3}$ graph which is also indicated in the figure. Unlike standard cacti, the branches of triangles eventually close to form large loops, since the underlying $\phi^{3}$ graphs are closed. For an alternative physical interpretation one could consider the $\phi^{3}$ graphs themselves and think of the model as a gas of $y$ loops in an $x$ "sea", which is the $O(1)$ loop gas representation of the Ising model.

Viewed as Ising spins on a cactus graph the action of equ.(77, 10) restricts triangles to have all spins up $\left(x^{3}\right.$ vertices $)$ or two spins down and one up $\left(x y^{2}\right.$ vertices). This restriction does not appear in the planar case as one can then think of $x$ representing a "same-spin" edge and $y$ a "different-spin" edge, which is sufficient to cover all configurations when the triangles are pasted together along their edges. The difference on the thin graphs is that the triangles are to be thought of as being joined at their vertices. Lifting the constraint is equivalent to allowing non-zero $\delta$ and $\beta$ in the original vertex model and we obtain actions that describe multi-spin interaction models on cacti. The spin Hamiltonian for such a model is given by

$$
H=\beta\left(J_{3} \sum_{\Delta} \sigma_{i} \sigma_{j} \sigma_{k}+h \sum_{i} \sigma_{i}\right)
$$

where the three spin sum is over triangles $\Delta$. With all four possible spin configurations on the triangles of the Husimi tree now allowed, namely $(+++),(---),(++-),(+--)$, the action can be written as

$$
S=\frac{\mu}{2} x^{2}+\frac{1}{2} y^{2}-\mu^{2} x^{2} y-\zeta \mu y^{2} x-\frac{\zeta \mu^{3}}{3} x^{3}-\frac{1}{3} y^{3},
$$

where $\mu=\exp (2 \beta h), \zeta=\exp \left(2 \beta J_{3}\right)$. This is clearly equivalent to equ.(6) once one rescales $x \rightarrow x / \sqrt{\mu}$ and makes the identifications

$$
\beta+3 \delta=1
$$




$$
\begin{aligned}
\alpha+3 \gamma & =\zeta \mu^{3 / 2} \\
\gamma-\alpha & =\zeta \mu^{1 / 2} \\
\delta-\beta & =\mu
\end{aligned}
$$

which gives two conditions on our original four vertex model couplings to match the number of physical parameters. Note that it is natural to include the external field $h$ from the start in such models as the phase transition generically appears in non-zero field for such multi-site interaction models [11].

The Hamiltonian of equ.(12) may be extended to include nearest neighbour interactions as well

$$
H=\beta\left(J_{3} \sum_{\Delta} \sigma_{i} \sigma_{j} \sigma_{k}+J_{2} \sum_{i j} \sigma_{i} \sigma_{j}+h \sum_{i} \sigma_{i}\right)
$$

and a similar reasoning to the pure 3 -spin interaction case above leads to the action

$$
S=\nu\left(\frac{\mu}{2} x^{2}+\frac{1}{2} y^{2}-\mu^{2} x^{2} y-\zeta \mu y^{2} x-\frac{\zeta \nu^{2} \mu^{3}}{3} x^{3}-\frac{\nu^{2}}{3} y^{3}\right),
$$

where the additional parameter $\nu=\exp \left(2 \beta J_{2}\right)$. Once again a rescaling $x \rightarrow x / \sqrt{\mu}$ and absorbing the overall factor of $\nu$ in the vertex coupling gives an action of the form equ.(6). We can also see that sending $J_{3} \rightarrow \infty(\zeta \rightarrow \infty)$ picks out the two sorts of vertex/triangle allowed in the Ising model discussed above. From the loop gas point of view both equ. (13, 16) represent a gas of $y$ loops and lines in an $x$ sea, which can now interact via $y^{3}$ terms and break via $x^{2} y$ terms. Alternatively the roles of $y$ (loops/lines) and $x$ (sea) may be exchanged as their vertices appear in a similar form in the action.

It is also possible to bring Potts models under the umbrella of the vertex model framework in one of two possible ways. The original $q$-state Potts model action contains $q$ (real) variables $\phi_{i}$

$$
S=\frac{1}{2} \sum_{i=1}^{q} \phi_{i}^{2}-c \sum_{i<j} \phi_{i} \phi_{j}-\frac{1}{3} \sum_{i=1}^{q} \phi_{i}^{3},
$$

where $c$ is now $1 /(\exp (2 \beta)+q-2)$, and represents the following Hamiltonian on $\phi^{3}$ graphs

$$
H=\beta \sum_{<i j>}\left(\delta_{\sigma_{i}, \sigma_{j}}-1\right)
$$

where the spins $\sigma_{i}$ take on $q$ values and $\delta$ is a Kronecker delta. It is possible to write an "effective" action coming from imposing the observed symmetry breaking pattern $\phi_{1 \ldots q-1}=x, \phi_{q}=y$ from the saddle point equation solutions on the original $q$ variable action [1]. This effective action

$$
S=\frac{1}{2}(q-1)[1-c(q-2)] x^{2}-\frac{1}{3}(q-1) x^{3}+\frac{1}{2} y^{2}-\frac{1}{3} y^{3}-c(q-1) x y
$$

faithfully reproduces the correct saddle point solutions. One can carry now out some rescalings on equ. 19] along with the ubiquitous linear transformation $x \rightarrow(x-y) / \sqrt{2}, y \rightarrow(x+y) / \sqrt{2}$ to obtain

$$
S=\frac{1}{2}\left(x^{2}+y^{2}\right)-\frac{1}{3}\left[x^{3}+3 \Omega \Delta x^{2} y+3 \Omega^{2} x y^{2}+\Omega^{3} \Delta y^{3}\right]
$$

where

$$
\begin{aligned}
\Omega & =\sqrt{\frac{\sqrt{\chi}-\kappa}{\sqrt{\chi}+\kappa}} \\
\chi & =(q-1)(1-c(q-2)), \quad \kappa=c(q-1) \\
\Delta & =\frac{(q-1)-\chi^{3 / 2}}{(q-1)+\chi^{3 / 2}}
\end{aligned}
$$


which is yet another variant of the action in equ.(6) for a particular choice of couplings. It is noteworthy that the first order transition point for the $q$-state Potts model that was derived in []

$$
c=\frac{1-(q-1)^{-1 / 3}}{q-2}
$$

is the point at which $\Delta=0$, reducing equ.(20) to an Ising-like action .

A second possibility is to include two further quadratic terms in the allowed action

$$
\begin{aligned}
\frac{1}{2}\left[\phi^{2}+\left(\phi^{\dagger}\right)^{2}\right] & =x^{2}-y^{2} \\
\frac{i}{2}\left[\phi^{2}-\left(\phi^{\dagger}\right)^{2}\right] & =-2 x y .
\end{aligned}
$$

which are best thought of as addition bivalent vertices from the point of view of maintaining a vertex model interpretation. These allow two arrow heads or tails to meet. With these additional vertices the action becomes

$$
\begin{aligned}
S & =\frac{1}{2}\left\{\mu \phi^{\dagger} \phi-(\nu+i \xi) \phi^{2}-(\nu-i \xi)\left(\phi^{\dagger}\right)^{2}\right. \\
& \left.-\frac{(\alpha-i \beta)}{3}\left(\phi^{\dagger}\right)^{3}-\frac{(\alpha+i \beta)}{3} \phi^{3}-(\gamma+i \delta)\left[\left(\phi^{\dagger}\right)^{2} \phi\right]-(\gamma-i \delta)\left[\phi^{2}\left(\phi^{\dagger}\right)\right]\right\}
\end{aligned}
$$

and choosing $\alpha=\gamma=(q-1) / 4, \beta=\delta=1 / 4, \xi=-c(q-1) / 2, \mu=[1+(q-1)(1-c(q-2))] / 2$, $\nu=[1-(q-1)(1-c(q-2))] / 4$ gives an action which is recognisable as that for a $q$-state Potts model in equ.(19) when written in real form.

To summarize the results of this section - the action of equ.(5) for a vertex model on thin $\phi^{3}$ graphs has been shown to contain as special cases Ising and multi-spin interaction models, results which are easiest to see once the model has been rewritten in the real form of equ.(6). Written is this form the vertex models can be re-interpreted as representing various systems, including spins on cacti or loop/line gases on the original $\phi^{3}$ graphs. Potts models may also be incorporated into the general framework, either by reconstructing the effective Potts model action by rescaling and linear transformations from equ.(6), or by introducing further quadratic vertices as in equ.(24).

\section{Vertex Models on (Thin) $\phi^{4}$ Graphs}

We deal with the $\phi^{4}$ vertex models rather more briefly, as the essential features are similar to the $\phi^{3}$ models in the previous section. For $\phi^{4}$ graphs one has the option of being able to preserve the in-degree and out-degree of each sort of vertex, but as we have noted this is equivalent to considering potentials composed of products of $\phi^{\dagger} \phi$ and exploring a rather restricted class of critical behaviours. In a similar manner to the $\phi^{3}$ graphs, we therefore consider all the conjugation symmetric terms

$$
\begin{aligned}
& {\left[\left(\phi^{\dagger}\right)^{4}+\phi^{4}\right], \quad i\left[\left(\phi^{4}-\left(\phi^{\dagger}\right)^{4}\right]\right.} \\
& {\left[\left(\phi^{\dagger}\right)^{2} \phi^{2}\right],} \\
& {\left[\left(\phi^{\dagger}\right)^{3} \phi+\phi^{3}\left(\phi^{\dagger}\right)\right], \quad i\left[\left(\phi^{\dagger}\right)^{3} \phi-\phi^{3}\left(\phi^{\dagger}\right)\right] .}
\end{aligned}
$$

The most general action containing all these terms is

$$
\begin{aligned}
S & =\frac{1}{2}\left\{\phi^{\dagger} \phi-\frac{\alpha}{4}\left[\left(\phi^{\dagger}\right)^{4}+\phi^{4}\right]-i \frac{\beta}{4}\left[\phi^{4}-\left(\phi^{\dagger}\right)^{4}\right]-\gamma\left(\phi^{\dagger}\right)^{2} \phi^{2}\right. \\
& \left.-\sigma\left[\left(\phi^{\dagger}\right)^{3} \phi+\phi^{3}\left(\phi^{\dagger}\right)\right]-i \rho\left[\left(\phi^{\dagger}\right)^{3} \phi-\phi^{3}\left(\phi^{\dagger}\right)\right]\right\}
\end{aligned}
$$

or

$$
S=\frac{1}{2}\left\{\phi^{\dagger} \phi-\frac{(\alpha+i \beta)}{4} \phi^{4}-\frac{(\alpha-i \beta)}{4}\left(\phi^{\dagger}\right)^{4}-\gamma\left(\phi^{\dagger}\right)^{2} \phi^{2}-(\sigma+i \rho)\left[\left(\phi^{\dagger}\right)^{3} \phi\right]-(\sigma-i \rho)\left[\phi^{3}\left(\phi^{\dagger}\right)\right]\right\} .
$$


As for the $\phi^{3}$ examples, this is best digested in bite-size chunks. Switching to real notation

$$
\begin{aligned}
S & =\frac{1}{2}\left(x^{2}+y^{2}\right)-\frac{(\alpha+2 \gamma+4 \sigma)}{4} x^{4}-\frac{(\alpha+2 \gamma-4 \sigma)}{4} y^{4}--\frac{(3 \alpha+2 \gamma)}{2} x^{2} y^{2} \\
& -(2 \rho+\beta) x^{3} y-(2 \rho-\beta) x y^{3}
\end{aligned}
$$

we again find an action that has Ising and multi-spin interaction critical behaviour for various reduced subsets of its coupling constants. Setting the asymmetric terms $\rho$ and $\beta$ to zero again gives mean-field Ising critical behaviour, as can be seen in an exactly analogous fashion to the $\phi^{3}$ graphs by carrying out the linear transformation $x \rightarrow \frac{1}{\sqrt{2}}(x+y), x \rightarrow \frac{1}{\sqrt{2}}(x-y)$ on the action for an Ising model living on $\phi^{4}$ graphs

$$
S=\frac{1}{2}\left(x^{2}+y^{2}\right)-c x y-\frac{1}{4}\left(x^{4}+y^{4}\right)
$$

to get (with suitable scalings)

$$
S=\frac{1}{2}\left(x^{2}+y^{2}\right)-\frac{1}{4} x^{4}-\frac{3}{2} c^{*} x^{2} y^{2}-\frac{1}{4}\left(c^{*}\right)^{2} y^{4} .
$$

The original $\phi^{4}$ action displays a mean-field Ising phase transition at $c=1 / 2$, so one would expect the transformed action to have a transition at $c^{*}=1 / 3$, which a direct solution of the saddle point equations for equ.(30)

$$
\begin{aligned}
& x=1, \quad y=0 \quad\left(\operatorname{LowT}^{*}\right) \\
& x=\frac{1}{2} \sqrt{\frac{3-c^{*}}{2 c^{*}}}, \quad y=\sqrt{\frac{3 c^{*}-1}{8\left(c^{*}\right)^{2}}} \quad\left(\text { HighT }^{*}\right)
\end{aligned}
$$

confirms, just as for the $\phi^{3}$ Ising model.

Once again the Ising-like actions are seen to reside in the symmetric subspace of couplings. Allowing non-zero $\rho$ and $\beta$ in equs. (26,28) gives actions that may be interpreted as representing either multi-spin interactions on cacti composed of squares, such as that shown in Fig.3, or loop/line gases on the original $\phi^{4}$ graphs. The spin model Hamiltonians on cacti graphs represented by such actions are of the form

$$
H=\beta\left(J_{4} \sum_{[i, j, k, l]} \sigma_{i} \sigma_{j} \sigma_{k} \sigma_{l}+J_{2} \sum_{i j} \sigma_{i} \sigma_{j}+h \sum_{i} \sigma_{i}\right),
$$

where the sum $[i, j, k, l]$ is round the squares of the cactus, with similar relations between the spin couplings $\left(J_{4}, J_{2}, h, \beta\right)$ and the coefficients in the action equ.(28) as in the $\phi^{3}$ case.

\section{Vertex Models on Planar (Fat) Graphs}

Writing down vertex model actions for the $N \rightarrow \infty$ limit is a simple matter of putting a $T r$ in front of the previously discussed actions such as equ.(5) and interpreting the variables as $N \times N$ matrices rather than

scalars. In the case of higher than $\phi^{3}$ potentials the non-commutativity of the matrix variables will also allow the introduction of new terms compared with the thin graphs as, for example, terms such as $\left[\Phi^{\dagger} \Phi\right]^{2}$ and $\left[\Phi^{\dagger}\right]^{2} \Phi^{2}$, where the $\Phi, \Phi^{\dagger}$ are now $N \times N$ matrices, may be distinguished. This difference from the thin graph case arises because a consistent cyclic order of legs round a vertex may be defined in the planar limit which makes such distinctions meaningful, as can be seen in Fig.4. At the level of the partition function an explicit contour integral to pick out the $2 n$th order is no longer necessary as the number of planar graphs increases only exponentially with the order, so the vertex coupling ("cosmological constant") may be tuned to get a diverging number of vertices. This also means that the factorial prefactor $N_{n}$ need no longer be peeled off. Writing down such partition functions is one thing, solving them is another as one is no longer dealing with saddle points in simple scalar integrals. In general the result is an insoluble, or at least unsolved, matrix model. Nonetheless, the planar graph vertex models can be mapped onto other, solved, models in some particular cases which we discuss below. 
The non-commutativity of the matrix variables in the planar fat graph limit $N \rightarrow \infty$ plays no role on $\phi^{3}$ graphs, as the cyclic symmetry of the trace ensures that possible potential terms are identical to the thin graph case. We have, writing $\Phi=X+i Y$ for the $N \times N$ complex matrix $\Phi$ and $N \times N$ Hermitian matrices $X, Y$

$$
\begin{aligned}
\operatorname{Tr}\left[\left(\Phi^{\dagger}\right)^{2} \Phi+\Phi^{2}\left(\Phi^{\dagger}\right)\right] & =2 \operatorname{Tr}\left[X^{3}+X Y^{2}\right] \\
i \operatorname{Tr}\left[\left(\Phi^{\dagger}\right)^{2} \Phi-\Phi^{2}\left(\Phi^{\dagger}\right)\right] & =2 \operatorname{Tr}\left[Y^{3}+Y X^{2}\right] \\
\operatorname{Tr}\left[\left(\Phi^{\dagger}\right)^{3}+\Phi^{3}\right] & =2 \operatorname{Tr}\left[X^{3}-3 X Y^{2}\right] \\
i \operatorname{Tr}\left[\Phi^{3}-\left(\Phi^{\dagger}\right)^{3}\right] & =2 \operatorname{Tr}\left[Y^{3}-3 Y X^{2}\right],
\end{aligned}
$$

just as for thin graphs. The fat graph version of the action in equs.(5).65) therefore differs only in the presence of a trace

$$
S=\operatorname{Tr}\left\{\frac{1}{2}\left(X^{2}+Y^{2}\right)-\frac{(\alpha+3 \gamma)}{3} X^{3}-\frac{(\beta+3 \delta)}{3} Y^{3}-(\gamma-\alpha) X Y^{2}-(\delta-\beta) X^{2} Y\right\} .
$$

Equ.(34) is known to represent the Ising model on dynamical triangulations (i.e. the dual of planar $\phi^{3}$ graphs) with the spins situated at the triangle vertices, when $\beta=\delta=0$ and $(\gamma-\alpha) /(\alpha+3 \gamma)=c^{*}$ [10]. Its critical behaviour is therefore that of the Ising model coupled to $2 \mathrm{D}$ gravity, giving the KPZ critical exponents 12] rather than the flat 2D lattice Onsager exponents. We can say little about the more general case, as the solution of the resulting matrix model is not known.

For fat $\phi^{4}$ graphs the non-commutativity does play a role as there are now more quartic invariants than in the thin case. Writing $\Phi=X+i Y$ again, we have the following possibilities

$$
\begin{aligned}
\operatorname{Tr}\left[\left(\Phi^{\dagger}\right)^{2} \Phi^{2}\right] & =\operatorname{Tr}\left[X^{4}+Y^{4}+2 X Y X Y\right] \\
\operatorname{Tr}\left[\left(\left(\Phi^{\dagger}\right) \Phi\right)^{2}\right] & =\operatorname{Tr}\left[X^{4}+Y^{4}+4 X^{2} Y^{2}-2 X Y X Y\right] \\
\operatorname{Tr}\left[\left(\Phi^{\dagger}\right)^{4}+\Phi^{4}\right] & =2 \operatorname{Tr}\left[X^{4}+Y^{4}-4 X^{2} Y^{2}-2 X Y X Y\right] \\
i \operatorname{Tr}\left[\Phi^{4}-\left(\Phi^{\dagger}\right)^{4}\right] & =8 \operatorname{Tr}\left[X Y^{3}-X^{3} Y\right] \\
\operatorname{Tr}\left[\left(\Phi^{\dagger}\right)^{3} \Phi+\Phi^{3}\left(\Phi^{\dagger}\right)\right] & =2 \operatorname{Tr}\left[X^{4}-Y^{4}\right] \\
i \operatorname{Tr}\left[\left(\Phi^{\dagger}\right)^{3} \Phi-\Phi^{3}\left(\Phi^{\dagger}\right)\right] & =4 \operatorname{Tr}\left[X^{3} Y+Y^{3} X\right] .
\end{aligned}
$$

Dropping the terms containing an asymmetric combination of $\Phi, \Phi^{\dagger}$ it is possible to construct an action that is equivalent to the Ising action for spins on the vertices of a random quadrangulation (i.e. the duals of $\phi^{4}$ graphs) by choosing the appropriate combination of the remaining terms

$$
S=\operatorname{Tr}\left\{\frac{1}{2} X^{2}+\frac{1}{2} Y^{2}-\frac{1}{4} X^{4}-\frac{c^{*}}{2}\left(X Y X Y+2 X^{2} Y^{2}\right)-\frac{\left(c^{*}\right)^{2}}{4} Y^{4}\right\},
$$

where $c^{*}$ is again the dual to to the Ising coupling $c=\exp (-2 \beta), c^{*}=(1-c) /(1+c)$. That equ.(36) does represent an Ising model can be shown by retracing the steps that lead from equ.(29) to equ.(30) in the thin graph case, or by deriving the weights in equ.(36) from direct consideration of the Ising model on the random quadrangulation [10]. The most general $\phi^{4}$ vertex model containing a linear combination of the terms in equ.(35) remains unsolved, but some further examples can be solved, or at least reduced to eigenvalue integrals and solved in principle.

One of these was noted some years ago in 13 by Ginsparg. If we consider the action

$$
S=\operatorname{Tr}\left\{\frac{1}{2} \Phi^{\dagger} \Phi-\alpha\left[\left(\Phi^{\dagger} \Phi\right)^{2}+\left(\Phi^{\dagger}\right)^{2} \Phi^{2}\right]\right\}
$$

the resulting Hermitian matrix model action is

$$
S=\operatorname{Tr}\left\{\frac{1}{2}\left(X^{2}+Y^{2}\right)-2 \alpha\left(X^{2}+Y^{2}\right)^{2}\right\}
$$


which can be seen to be an $O(2) / X Y$ model by introducing a further Hermitian matrix $M$ and rewriting the potential term to give

$$
S=\operatorname{Tr}\left\{\frac{1}{2}\left(X^{2}+Y^{2}+M^{2}\right)-2 \sqrt{\alpha}\left(X^{2}+Y^{2}\right) M\right\} .
$$

Allowing oneself the luxury of two couplings rather than the one of the $X Y$ model also gives rise to an interesting class of models. The combination

$$
S=\operatorname{Tr}\left\{\frac{1}{2} \Phi^{\dagger} \Phi-\alpha\left(\Phi^{\dagger}\right)^{2} \Phi^{2}-\beta\left[\left(\Phi^{\dagger} \Phi\right)^{2}\right]\right\}
$$

which is equivalent to the real action

$$
S=\operatorname{Tr}\left\{\frac{1}{2}\left(X^{2}+Y^{2}\right)-(\alpha+\beta)\left(X^{4}+Y^{4}\right)-2(\alpha-\beta) X Y X Y-4 \beta X^{2} Y^{2}\right\}
$$

can be shown to be a free fermion model 14 .

Taking another combination

$$
S=\operatorname{Tr}\left\{\frac{1}{2} \Phi^{\dagger} \Phi-\alpha\left(\Phi^{\dagger}\right)^{2} \Phi^{2}-\frac{\beta}{2}\left[\left(\Phi^{\dagger}\right)^{4}+\Phi^{4}\right]\right\}
$$

gives

$$
S=\operatorname{Tr}\left\{\frac{1}{2}\left(X^{2}+Y^{2}\right)-(\alpha+\beta)\left(X^{4}+Y^{4}\right)-2(\alpha-\beta) X Y X Y+4 \beta X^{2} Y^{2}\right\}
$$

which is similar to the free fermion model apart from the sign of the $X^{2} Y^{2}$ coupling.

If we take $\beta$ negative and $\alpha=-\beta$ in equation.(43) we get the action

$$
S=\operatorname{Tr}\left\{\frac{1}{2}\left(X^{2}+Y^{2}\right)-4|\beta|\left(X Y X Y+X^{2} Y^{2}\right)\right\}
$$

considered by Cicuta et.al. [15] in their discussion of a three-edge colouring problem for planar graphs. Although not yet solved exactly the partition function for this model can be reduced to an eigenvalue integral and its properties explored

$$
Z=\int \prod_{i} d \lambda_{i} \prod_{i<j}\left(\lambda_{i}-\lambda_{j}\right)^{2} \exp \left(-\frac{1}{2} \sum_{i} \lambda_{i}^{2}\right) \prod_{i j}\left(1-4|\beta|\left(\lambda_{i}+\lambda_{j}\right)^{2}\right)^{-\frac{1}{2}}
$$

where the $\lambda_{i}$ are the $N$ eigenvalues of the $N \times N$ matrices. The scaling of the edge of the eigenvalue distribution of this model in the $N \rightarrow \infty$ limit suggest that the critical behaviour is Ising-like, as the exponents are identical.

The same eigenvalue expression arises on taking $\alpha=\beta$ ( $\beta$ positive) in equation.(13) to give the action

$$
S=\operatorname{Tr}\left\{\frac{1}{2}\left(X^{2}+Y^{2}\right)-2 \beta\left(X^{2}-Y^{2}\right)^{2}\right\}
$$

Adopting similar tactics to the $X Y$ model and introducing a further matrix $M$ gives

$$
S=\operatorname{Tr}\left\{\frac{1}{2}\left(X^{2}+Y^{2}+M^{2}\right)-2 \sqrt{\beta}\left(X^{2}-Y^{2}\right) M\right\}
$$

or

$$
S=\operatorname{Tr}\left\{\frac{1}{2} M^{2}+\frac{1}{2} X^{2}(1-2 \sqrt{\beta} M)+\frac{1}{2} Y^{2}(1+2 \sqrt{\beta} M)\right\}
$$


whose partition function reduces to the eigenvalue integral

$$
Z=\int \prod_{i} d \lambda_{i} \prod_{i<j}\left(\lambda_{i}-\lambda_{j}\right)^{2} \exp \left(-\frac{1}{2} \sum_{i} \lambda_{i}^{2}\right) \prod_{i j}\left(1-2 \sqrt{\beta}\left(\lambda_{i}+\lambda_{j}\right)\right)^{-\frac{1}{2}}\left(1+2 \sqrt{\beta}\left(\lambda_{i}+\lambda_{j}\right)\right)^{-\frac{1}{2}}
$$

which is identical to equation.(45) when the two measure factors are combined. This is not so surprising when one realizes that the actions of equ.(44) and equ.(46) are related by our old friend the linear transformation $X \rightarrow(X+Y) / \sqrt{2}, Y \rightarrow(X-Y) / \sqrt{2}$.

We note in closing that the appropriate linear combination of the first three terms in equ.(35) produces an $X Y X Y$ potential alone, which was also shown in [16] to display Ising critical behaviour by carrying out an ingenious non-linear transformation on the matrices. Similar models occur in the enumeration of meanders [17].

\section{Discussion}

We have considered vertex models on thin $\phi^{3}$ and $\phi^{4}$ graphs and shown that various sorts of critical behaviour - Ising, Potts, multi-spin interaction, ... may be obtained by restricting the most general couplings allowed by the arrow reversal symmetry in the vertex models to various subsets. In this sense the vertex models discussed here play a similar role to the eight vertex model on the regular square lattice, in whose phase diagram lurks the critical behaviour of many other familiar models [9]. One important difference from the regular lattice case is that the notion of orientational order for edges round a vertex is lost on random graphs (there is no "up" and "down"). We showed that writing the vertex model actions in real form allowed us to recognise transformations from other known models and deduce the critical behaviour. The simplicity of the thin graph models also allowed the explicit solution of the saddle point equations in some cases to confirm these deductions, in particular for the various Ising models. We noted that the vertex models, when written in their equivalent real form, also admitted a physical interpretation in terms of spin models on cacti graphs, or loop/line gases on the original random graphs.

Although just as easy to write down actions for fat graphs, solving such models is a rather more difficult task than on thin graphs. We presented some solutions for vertex models on fat graphs by showing their equivalence to already solved models, mostly by following a similar tack to the thin graph exposition and rewriting the models in terms of two Hermitian matrices. In these examples Ising, XY and Ising-like (for the model of [15]) critical behaviour was observed, as well as a model that was equivalent to free fermions (that of equ.(41), 14 ). On fat graphs the cyclic order of edges round a vertex may be consistently defined, so the number of distinguishable terms in higher than $\phi^{3}$ potentials is greater than in the thin graph case.

As noted in the introduction, the idea of using integrals over complex variables and saddle point methods to extract the asymptotic behaviour of directed graph models for large graphs has been suggested by Whittle [6]. From a graph theory point of view all of the calculations in this paper are exercises in counting various sorts of more or less esoteric directed random graphs. The use of scalar "actions" and saddle point methods seems to be as effective in investigating the statistical mechanics of vertex models on thin graphs (or, if one prefers, the statistical mechanics of directed graphs) as similar methods have been for other statistical mechanical, notably spin, models living on thin graphs.

DAJ would like to thank Thordur Jonsson for various discussions about vertex models on planar graphs.

\section{References}

[1] D. Johnston and P. Plecháč, "Potts Models on Feynman Diagrams", hhep-lat/9704020;

D. Johnston and P. Plecháč, "Percolation on a Feynman Diagram", cond-mat/9705101;

D. Johnston and P. Plecháč, "Why Loops Don't Matter", cond-mat/9705101.

[2] C. Baillie, D. Johnston and J-P. Kownacki, Nucl. Phys. B432 (1994) 551;

C. Baillie, W. Janke, D. Johnston and P. Plecháč, Nucl. Phys. B450 (1995) 730; 
C. Baillie and D. Johnston, Nucl. Phys. B47 (Proc. Suppl.) (1996) 649;

C. Baillie, D. Johnston, E. Marinari and C. Naitza, J. Phys. A29 (1996) 6683;

C. Baillie, N. Dorey, W. Janke and D. Johnston, Phys. Lett B369 (1996) 123.

[3] C. Bachas, C. de Calan and P. Petropoulos, J. Phys. A27 (1994) 6121.

[4] T. Banks, C. Bender, T-T. Wu, Phys. Rev. D8 (1973) 3346;

T. Banks, C. Bender, Phys. Rev. D8 (1973) 3367;

E. Brezin, J. Le Guillou and J. Zinn-Justin, Phys. Rev. D15 (1977) 1544;ibid 1558;

G. Parisi, Phys. Lett. 66B (1977) 167;

N. Lipatov, JETP Lett. 24 (1976) 157; Sov. Phys. JETP 44 (1976) 1055; JETP Lett. 25 (1977) 104;

Sov. Phys. JETP 45 (1977) 216;

S . Coleman, Phys. Rev. D15 (1977) 2929;

C. Callan and S. Coleman, Phys. Rev. D16 (1977)

[5] P. Whittle, Adv. Appl. Prob. 24 (1992) 455;

in Disorder in Physical Systems, ed. G.R. Grimmett and D.Welsh, (1990) 337.

[6] P. Whittle, J. Stat. Phys. 56 (1989) 499.

[7] T. Morris, Nucl. Phys. B356 (1991) 703.

[8] W. Janke, Phys. Lett. A143 (1990) 107.

[9] R. Baxter, "Exactly Soluble Models in Statistical Mechanics", Academic Press, London, 1982.

[10] D. Johnston, Phys. Lett. B314 (1993) 69;

C. Baillie and D. Johnston, Phys. Lett. B357 (1995) 287;

S. Carroll, M. Ortiz and W. Taylor, Nucl. Phys. B468 (1996) 620.

[11] X. Wu and F. Wu, J. Phys. A 22 (1989) L1031;

R. Baxter and F. Wu, Phys. Rev. Lett 31 (1973) 1294;

C. Thompson, J. Stat. Phys. 27 (1982) 441; ibid 457;

J. Monroe, J.Stat. Phys. 65 (1991) 255; ibid 67 (1992) 1185;

P Gujrati, Phys. Rev. Lett. 74 (1995) 809;

A. Ananikian, S. Dallakian, N. Izmailian and K. Oganessyan, Phys. Lett. A214 (1996) 205;

A. Alahverdian, N. Ananikian, S. Dallakian, "Singularities at a Dense Set of Temperature in Husimi Tree", cond-mat/9702106.

[12] V.G. Knizhnik, A.M. Polyakov and A.B. Zamolodchikov, Mod. Phys. Lett. A3 (1988) 819.

F. David, Mod. Phys. Lett. A3 (1988) 1651.

J. Distler and H. Kawai, Nucl. Phys. B321 (1989) 509.

[13] P. Ginsparg, "Matrix Models and 2D Gravity" hep-th/9112013], also in Phys. Rep. 254 (1995).

[14] S. Dalley, Mod. Phys. Lett A7 (1992) 1651.

[15] G. Cicuta, L. Molinari and E. Montaldi, Phys. Lett. B306 (1993) 245.

[16] L. Chekhov and C. Kristjansen, Nucl. Phys. B479 (1996) 683.

[17] O. Golinelli and E. Guitter, Nucl. Phys. B482 (1996) 497. 
(a)
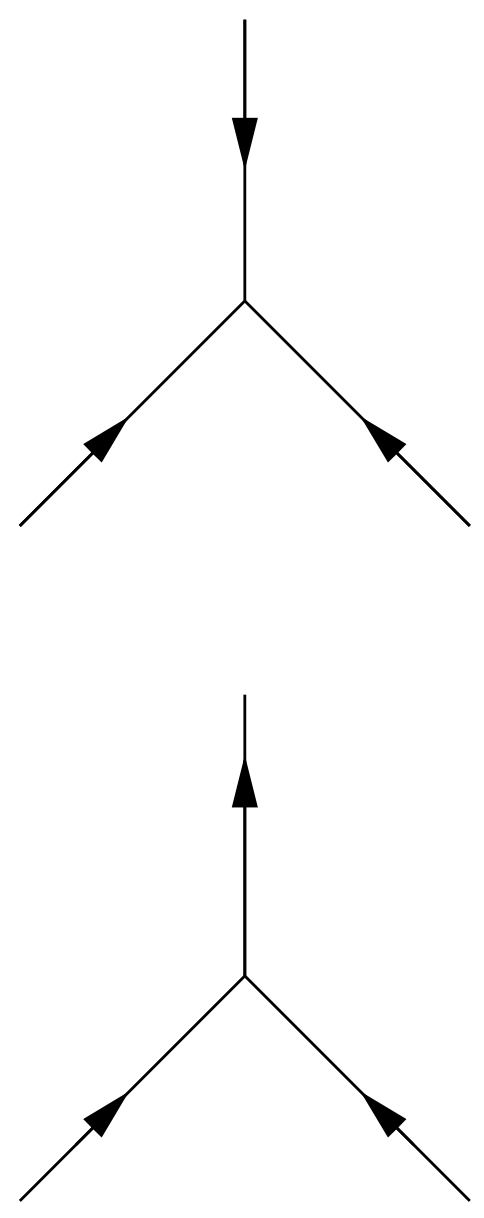

(c) (b)
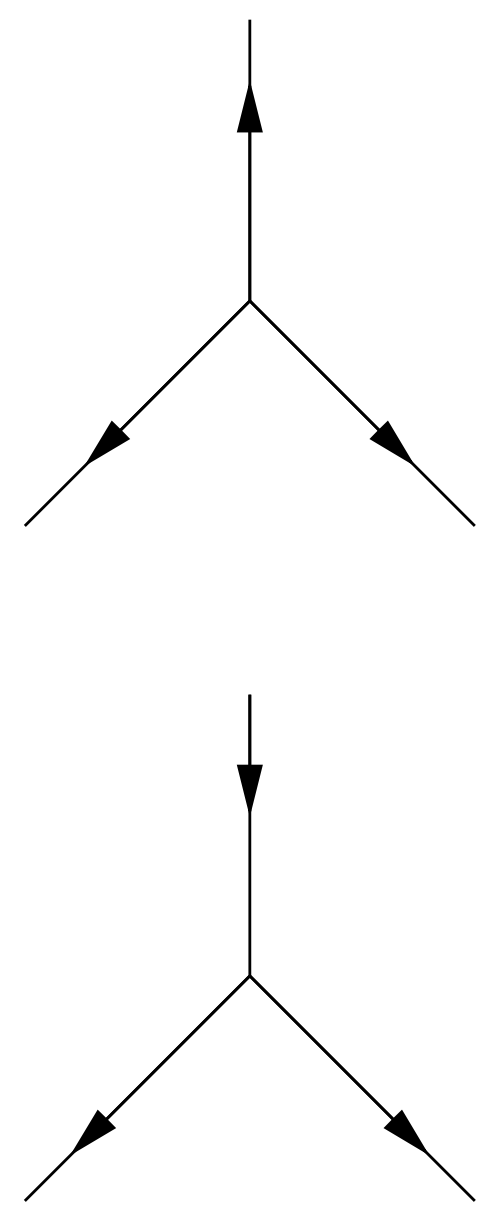

(d)

Figure 1: The various sorts of $\phi^{3}$ vertices which appear in the model:(a) $\left(\phi^{\dagger}\right)^{3}$, (b) $\phi^{3},(\mathrm{c})\left(\phi^{\dagger}\right)^{2} \phi$, (d) $\phi^{2} \phi^{\dagger}$ 


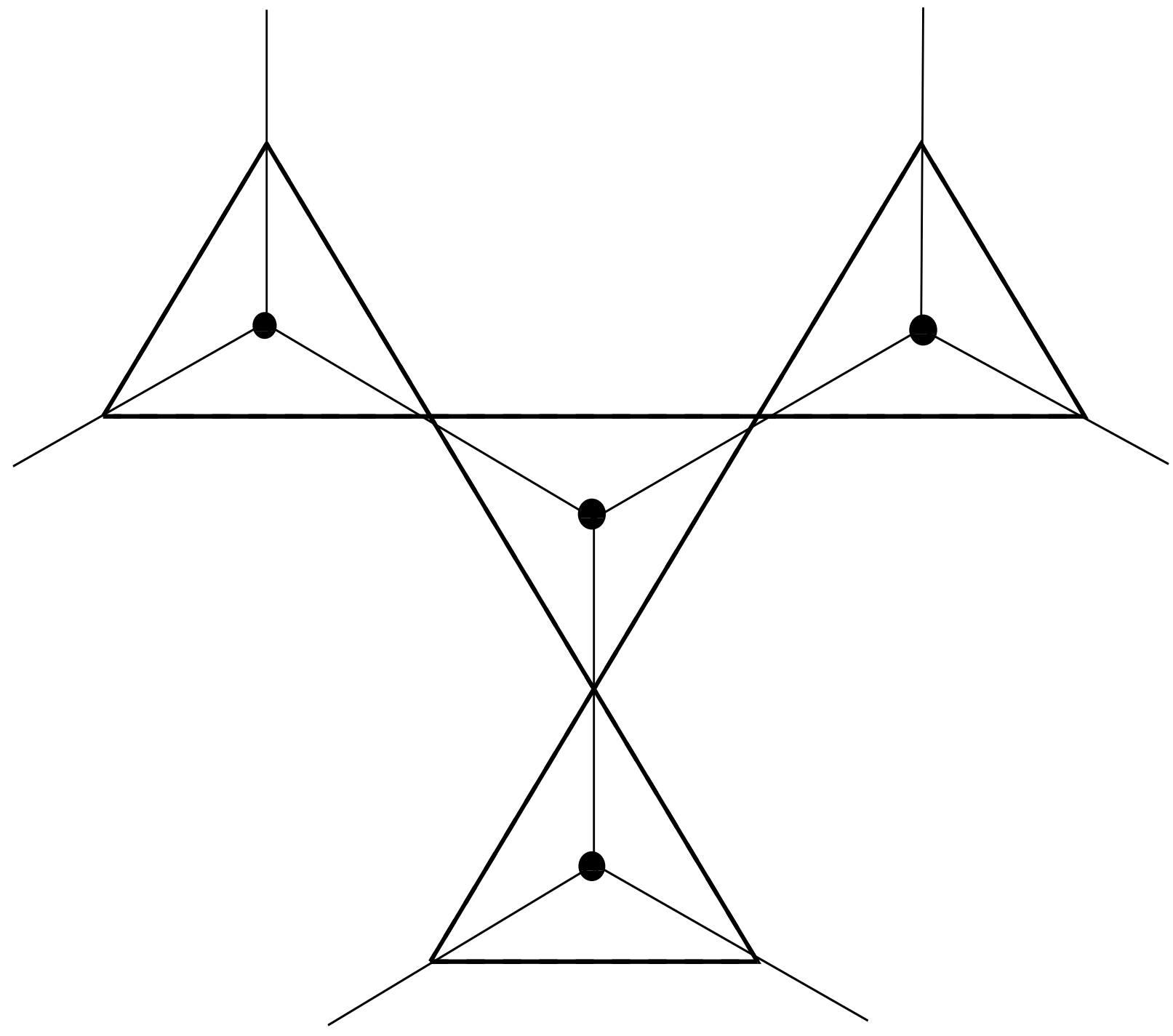

Figure 2: A section of a Husimi tree composed of triangles. The underlying $\phi^{3}$ graph is also shown. 


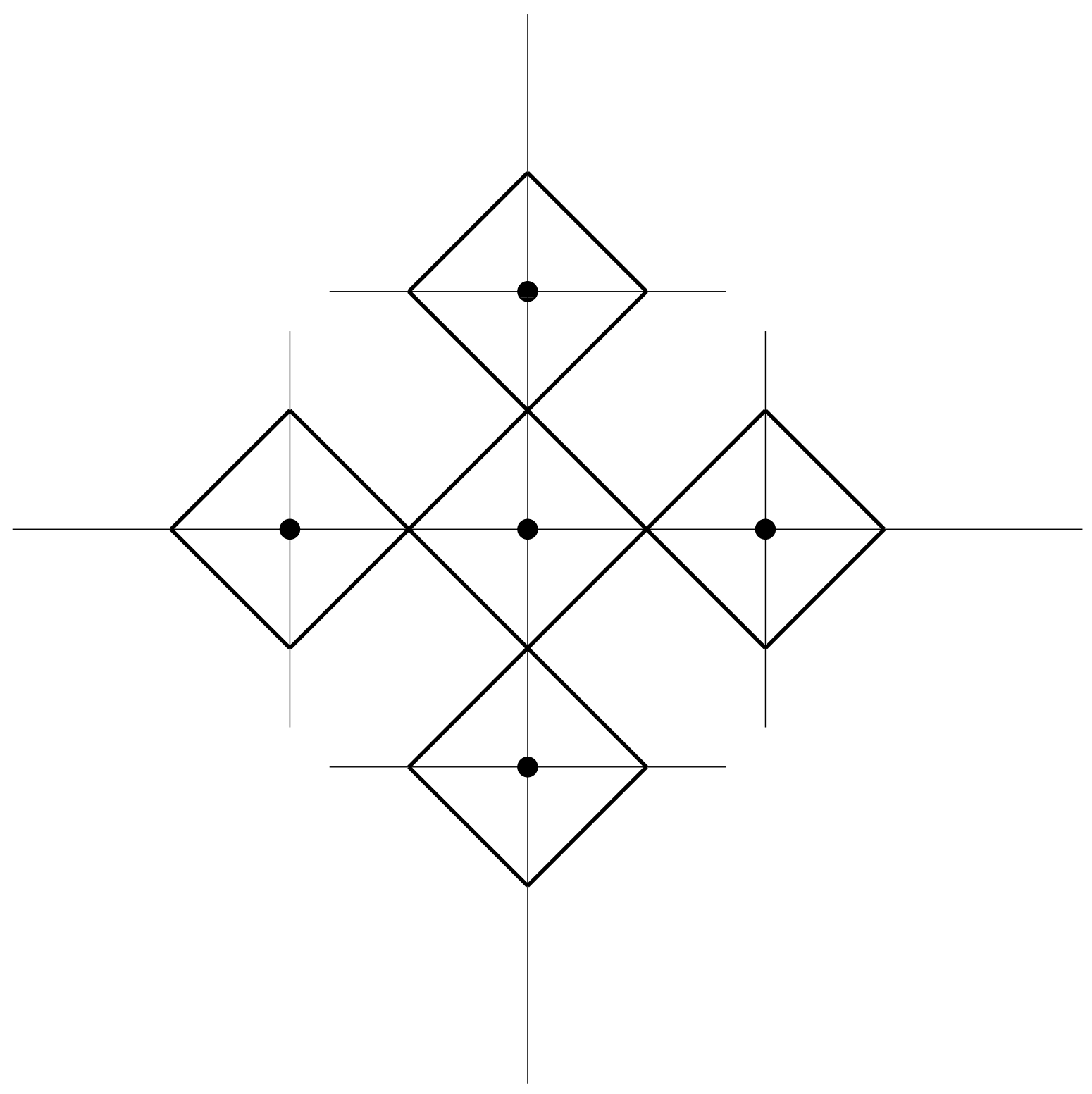

Figure 3: A section of a Husimi tree composed of squares. The underlying $\phi^{4}$ graph is also shown. 


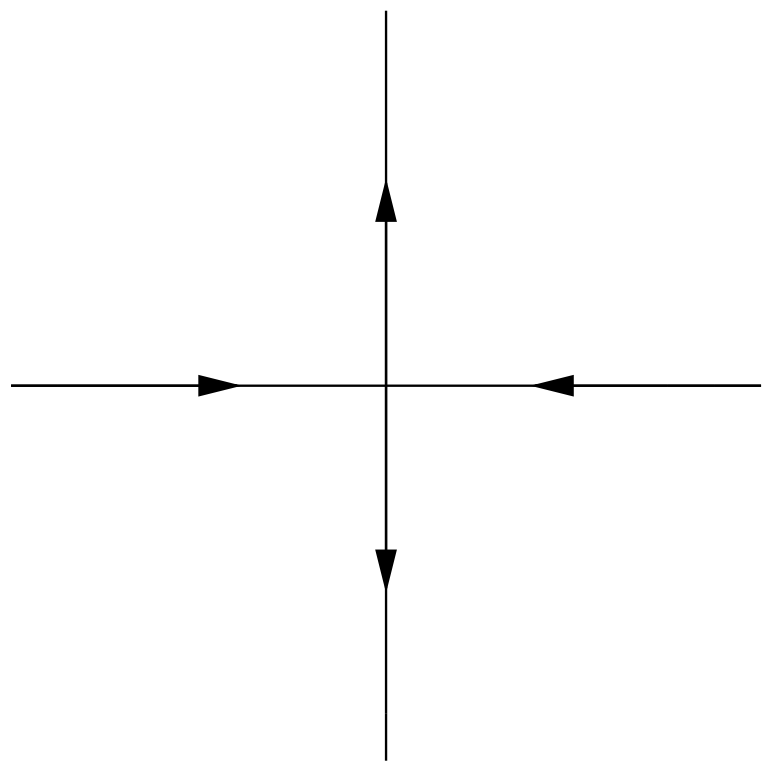

(a)

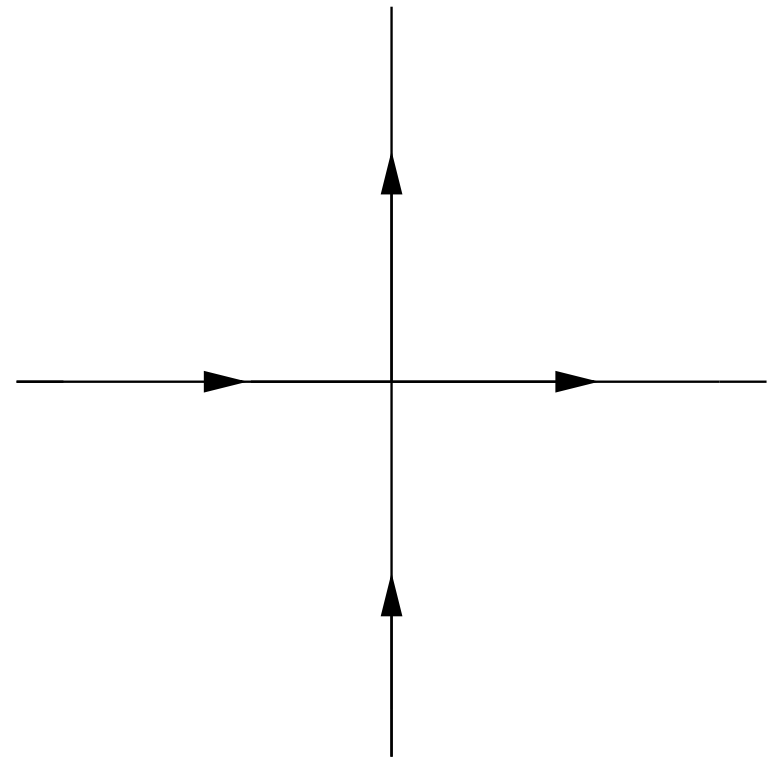

(b)

Figure 4: Two different cyclic orientations of a quartic vertex which may be distinguished in a planar graph: (a) $\left[\Phi^{\dagger} \Phi\right]^{2}$, (b) $\left[\Phi^{\dagger}\right]^{2} \Phi^{2}$ 